\title{
High-Throughput Tertiary Amine Deoxygenated Photopolymerizations for Synthesizing Polymer Libraries
}

\author{
Christopher Stubbs, ${ }^{\dagger}$ Thomas Congdon, $^{\dagger}$ Jessica Davis, $^{\dagger}$ Daniel Lester, ${ }^{\dagger}$ Sarah-Jane Richards, ${ }^{\dagger}$
} and Matthew I. Gibson*, $*$,

${ }^{\dagger}$ Department of Chemistry and ${ }^{\star}$ Warwick Medical School, University of Warwick, Gibbet Hill Road, Coventry CV4 7AL, U.K.

\author{
Supporting Information
}

\begin{abstract}
The huge chemical space potential of synthetic polymers means that in many studies only a small parameter range can be realistically synthesized in a short time and hence the "best" formulations may not be optimum. Throughput is traditionally limited by the need for deoxygenation in radical polymerizations, but advances in photopolymerization now provide opportunities for "in-air" polymerizations. Here, we have developed a protocol using liquid handling robots (or multichannel pipettes) with blue light photolysis of reversible addition fragmentation chain transfer agents and tertiary amine deoxygenation to enable the synthesis of polymer

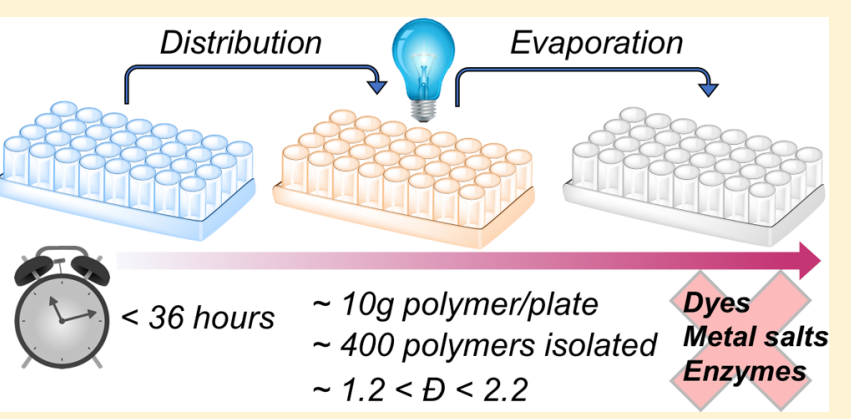
libraries in industry-standard 96 -well plates with no specialized infrastructure and no degassing step. The roles of solvents and amine deoxygenators are explored to optimize the polymerization, particularly to look at alternatives to dimethyl sulfoxide (DMSO) for hydrophobic monomer (co)polymerization. DMSO is shown to aid the degassing process but is not easy to remove and hence prevents isolation of pure polymers. In contrast, using dioxane in-plate evaporation or precipitation of the tertiary amine allowed isolation of polymers in-plate. This removed all reaction components yielding pure polymers, which is not easily achieved with systems using metal catalysts and secondary reductants, such as ascorbic acid. As an example of the throughput, in just under $40 \mathrm{~h}, 392$ polymers were synthesized and subsequently analyzed direct from plates by a 96-well plate sampling size exclusion chromatography system to demonstrate reproducibility. Due to less efficient degassing in dioxane (compared to DMSO), the molecular weight and dispersity control were limited in some cases (with acrylates giving the lowest dispersities), but the key aim of this system is to access hundreds of polymers quickly and in a format needed to enable testing. This method enables easy exploration of chemical space and development of screening libraries to identify hits for further study using precision polymerization methods.
\end{abstract}

\section{INTRODUCTION}

To match the complex function and structures of biomacromolecules (proteins, nucleic acids, polysaccharides, and polyketides) using synthetic materials, it is essential to incorporate increased diversity of monomer components to generate copolymers of increasing complexity. ${ }^{1-4}$ For example, recent advances in sequence-controlled ${ }^{5,6}$ and multiblock ${ }^{7,8}$ copolymers have led to emergent biological and biomimetic functions, and there is a need to explore polymer formulation space for many pharmaceutical applications. ${ }^{9}$ However, there is a major challenge in the huge chemical space to be explored, which scales dramatically when comonomers are used. As a result of this, high-throughput methods are required for material discovery and to elucidate structure-activity relationships between materials and their properties. ${ }^{10-13}$ Despite the ease and convenience of modern controlled radical polymerization (CRP) methods (i.e., reversible addition fragmentation chain transfer (RAFT) and atom transfer radical polymerization (ATRP)), reagent addition, degassing and crucially the isolation, and purification and transfer of the polymers into assay-ready formats are still bottlenecks for translation to truly high-throughput systems. Polymer investigations typically use panels of tens of polymers, which represents a very small proportion of the investigable chemical space. This means that specific formulations with emergent properties are often missed, and the sample size is very small when compared to commercially available standard libraries, for example, drug discovery or biological screening.

Schubert and co-workers reported protocols for the highthroughput synthesis of multiblock copolymers using RAFT polymerization, initiated thermally by an azo initiator. The system required the synthesizer to be placed in an inert environment and that all reagents should be kept under a constant flow of nitrogen. ${ }^{14}$ Destarac reported the use of a parallel synthesizer to prepare various acrylate copolymers via a MADIX process. $^{15}$ However, these systems require infrastructures to enable closed-to-air preparations that are not

Received: August 15, 2019

Published: October 2, 2019 
always available. Inert high-throughput techniques have also been applied using a variety of controlled radical techniques including ATRP and cationic ring opening polymerization (CROP). ${ }^{16,17}$ These strategies using robotic methods in inert atmospheres have also been reviewed by Schubert. ${ }^{18}$ Alexander and co-workers have exploited robotics to print diverse libraries of cross-linked copolymers onto surfaces to discover new materials to resist bacterial fouling ${ }^{19}$ or for stem cell expansion. ${ }^{11}$ Langer and co-workers have used step-growth polymerization methods to explore large (140 polymers) chemical space to identify gene transfection vectors. The advantage of this method is that only the solvent needs to be removed at the end of the reaction. A high-throughput screen was successful in identifying copolymers for solubilization of low-solubility pharmaceuticals, which could not have been easily achieved by rational design alone. ${ }^{20}$

When screening polymers for biological applications, it is essential that the polymers can be easily transferred into the format needed for testing, especially multiwell plates used in most biological testing. Otherwise, manual handling and weighing of hundreds of polymers create a process bottleneck. Ideally, in the prototype stage, reactions should be conducted in the multiwell plate format enabling direct transfer from the synthetic medium to the testing. ${ }^{21,22}$ There has been considerable recent interest in the development of oxygentolerant or intrinsically oxygen-depleting polymerization methods. One example employed enzymatic degassing; glucose oxidase $(\mathrm{GOx})$ was used to remove oxygen in the polymerization of hydroxyethyl acrylate, generating narrow dispersity polymers in methanol/PBS solutions. ${ }^{23}$ These materials reached conversions of $>90 \%$ in $3 \mathrm{~h}$ and when paired with a liquid handling robot allowed controlled, high-throughput synthesis of polymers from a variety of water soluble monomers $^{24}$ and in complex solvent mixtures. ${ }^{25}$ This GOx strategy has also been exploited by Matyjaszewski to enable oxygen-tolerant ATRP. ${ }^{26}$ However, separating the enzyme from the product polymers is nontrivial, and it does not work in organic solvents, which are essential if hydrophobic (co) monomers are to be used. An early report of using light to initiate a controlled living radical polymerization was reported by Hawker and co-workers in 2012. Using an iridium catalyst, excellent control over the polymerization of methyl methacrylate was achieved, demonstrating that propagation occurs only when the light is switched on, and the chain remains dormant without this external stimulus. ${ }^{27}$ Boyer and coworkers expanded the scope of potential monomers compatible with oxygen tolerant polymerization through the development of PET-RAFT (photoinduced electron transfer, reversible addition fragmentation chain transfer), which allows the polymerization of a wide variety of hydrophobic and hydrophilic monomers. ${ }^{28-30}$ This process involves the use of a photocatalyst to reduce the chain transfer agent, generating a radical species that can then initiate the polymerization. This technique was applied by Boyer et al. for the high-throughput synthesis of a range of functionalized linear polymers as well as more complex three-arm and four-arm star polymers. ${ }^{29}$ The polymerization of less activated monomers has been demonstrated via this technique, with the iridium-catalyzed polymerization of vinyl acetate shown to proceed equally well in both degassed and oxygen-rich conditions. ${ }^{31}$ Boyer et al. also pioneered the development of an oxygen-tolerant, enzyme-free technique for the synthesis of complex polymer architectures in aqueous media using eosin $\mathrm{Y}$ and ascorbic acid.
The applicability of this technique with high-throughput polymerization was also demonstrated, carrying out reactions in 96-well plates at a range of ultralow volumes for the synthesis of arm-first star polymers, ${ }^{30}$ and has been reviewed in detail. $^{32}$ Photopolymerization is also emerging as a tool to enable hybrid materials containing proteins (that could be sensitive to traditional thermal polymerization). ${ }^{33-35}$ The multifunctional nature of these photocatalysts allows a variety of mechanisms to be exploited. Sumerlin et al. investigated the possible reaction mechanisms involved in aqueous PET-RAFT polymerizations using eosin $\mathrm{Y}$ by varying the intensity and color of the light source used to initiate the polymerization, as well as in the presence and absence of a tertiary amine. Under different irradiation conditions, the photocatalyst could be either oxidized or reduced, leading to different initiation and reversible termination steps. When no photocatalyst was present, the trithiocarbonate initiates via direct photolysis to generate an initiating species that could then also act as the chain transfer agent. ${ }^{36-38}$

One alternative to these PET-RAFT strategies was introduced by Qiao and co-workers. It involves the use of a sacrificial tertiary amine to reduce a photoirradiated RAFT agent, which in turn converts dissolved oxygen to superoxide. The formed superoxide is hypothesized to be irreversibly trapped by reacting with DMSO in the system. ${ }^{39}$ This system is appealing due to its simplicity and there being no need for additional photocatalysts or enzymes. Crucially, it should be possible to remove all non-polymer attached components of this system under vacuum, unlike with metal catalysts where purification is a challenge. Boyer et al. have also used tertiary amines in their photoirradiated systems, noting a significant increase in rate of propagation when present. ${ }^{28}$ Qiao's method was then adapted for the screening of cationic polymers and their derivatives as antimicrobials. ${ }^{40}$ One downside to this strategy is the need for DMSO in the reaction mixture. DMSO has a high boiling point $\left(189^{\circ} \mathrm{C}\right)$, and hence, polymers need to be either precipitated, which is not always easy in highthroughput polymerization and may require several different solvent systems or prepared sufficiently concentrated for dilution at the point of assaying for function. ${ }^{41,42}$ Hemocompatibility (blood toxicity) assays, for example, require a final DMSO concentration typically below $1 \%{ }^{43}$ to avoid false negatives, and hence, alternatives to DMSO as the screening solvent are desirable.

Considering this, we wanted to explore the limits and scope of the tertiary amine degassing method for high-throughput combinatorial polymer synthesis as it is conceptually simple and crucially does not involve the addition of photoactive components (other than the RAFT agent, which is incorporated into polymer) that may interfere with downstream assays.

This manuscript reports a DMSO-free photopolymerization system that when paired with a liquid handling system enables the generation of polymer libraries in multiwell plates. Crucially, by adapting the solvent conditions, we enable inplate parallel purification and easy vacuum removal of solvent. To demonstrate the utility of this technique, a library of almost 400 polymers was synthesized in $<40 \mathrm{~h}$ within microplates using liquid handling systems. This system allows access to a wide variety of polymers with little processing and high recoveries $(\sim 10 \mathrm{~g}$ per plate). This does not allow for full control over molecular weight or dispersity but enables an easy route to obtain large compositional diversity with no specific 
Table 1. Composition of each Stock Solution Used in the Polymerizations Carried Out to Determine Monomer and Solvent Versatility

\begin{tabular}{|c|c|c|c|c|c|c|c|}
\hline \multirow[b]{2}{*}{ monomer } & \multirow[b]{2}{*}{ solvent } & \multicolumn{2}{|c|}{ monomer reservoir } & \multicolumn{4}{|c|}{ solvent reservoir } \\
\hline & & monomer $(\mathrm{mL})$ & volume added $(\mu \mathrm{L})$ & TEOA $(\mathrm{g})$ & solvent $(\mathrm{mL})$ & CTA $(\mu \mathrm{L})$ & volume added $(\mu \mathrm{L})$ \\
\hline \multirow[t]{2}{*}{ DMAEMA } & $\mathrm{MeOH}$ & 25 & 210 & 9.47 & 8.83 & 144 & 290 \\
\hline & dioxane & 25 & 150 & 8.91 & 12.96 & 103 & 350 \\
\hline \multirow[t]{2}{*}{ MA } & $\mathrm{MeOH}$ & 25 & 146 & 9.44 & 12.61 & 188 & 354 \\
\hline & dioxane & 25 & 94 & 9.34 & 15.92 & 121 & 406 \\
\hline HEAm & $\mathrm{MeOH} / \mathrm{Tol}$ & 50 & 108 & 18.63 & 30.21 & 242 & 392 \\
\hline
\end{tabular}

infrastructure required to generate new lead materials for further study. We discuss the limitations of this technique in respect to monomer selectivity and polymer characteristics.

\section{EXPERIMENTAL SECTION}

Materials. N-Hydroxyethyl acrylamide (97\%), methyl methacrylate (99\%), methyl acrylate (99\%), $N$-isopropyl acrylamide (97\%), $N$ isopropylmethacrylamide (97\%), and 2-(dimethylamino) ethyl methacrylate were purchased from Sigma-Aldrich and filtered through a plug of basic alumina to remove inhibitors prior to use. Triethanolamine (TEOA) (98\%), triethylamine (TEA) (>99.5\%), 2-cyano-2-propyl dodecyl trithiocarbonate, and all solvents were purchased from Sigma-Aldrich and used without further purification.

Physical and Analytical Methods. ${ }^{1} \mathrm{H}$ NMR spectra were recorded on Bruker DPX-300 and DPX-400 spectrometers using deuterated solvents purchased from Sigma-Aldrich. Chemical shifts are reported relative to residual nondeuterated solvents. Infrared data was recorded on a Bruker Vector 22 GI003097.

Size exclusion chromatography (SEC) was carried out in dimethylformamide (DMF) and run on an Agilent 1260 Infinity II MDS instrument equipped with differential refractive index (DRI), viscometry (VS), light scattering (LS), and variable wavelength UV detectors. The system was equipped with $2 \times$ PLgel Mixed D columns $(300 \times 7.5 \mathrm{~mm})$ and a PLgel $5 \mu \mathrm{m}$ guard column. The eluent is DMF with $5 \mathrm{mM} \mathrm{NH}_{4} \mathrm{BF}_{4}$ additive. Samples were run at $1 \mathrm{~mL} \mathrm{~min}^{-1}$ at 50 ${ }^{\circ} \mathrm{C}$. Poly(methyl methacrylate) (Agilent EasiVial) was used to create a third-order calibration between 500-900,000 Da for calibration. Analyte samples were filtered through a nylon filter with $0.22 \mu \mathrm{m}$ pore size before injection. Experimental molar mass $\left(M_{\mathrm{n}, \mathrm{SEC}}\right)$ and dispersity $(\boxplus)$ values of synthesized polymers were determined by conventional calibration using Agilent GPC/SEC software. High-throughput SEC was carried out on a PL SEC 50 Plus with a differential refractive index (DRI) detector. The system was equipped with either PL Rapide M $(7.5 \times 150 \mathrm{~mm})$, PL Rapide F $(10 \times 100 \mathrm{~mm})$ columns or $2 \times$ PL Rapide M $(7.5 \times 150 \mathrm{~mm})$ columns. The eluent is DMF with $1 \% \mathrm{LiBr}$ as the additive. Samples were run at $50{ }^{\circ} \mathrm{C}$ at either 2 or 1 $\mathrm{mL} \min ^{-1}$ based on a column set. Poly(methyl methacrylate) standards (Agilent EasiVial) were used for calibration to create a third-order calibration between 500-1,000,000 Da. Experimental molar mass $\left(M_{\mathrm{n}, \mathrm{SEC}}\right)$ and dispersity $(\nexists)$ values of synthesized polymers were determined by conventional calibration using Agilent GPC/SEC software. Polymers were prepared using a Gilson Pipette Max 268, with 200 and $20 \mu \mathrm{L}$ pipette heads.

In-Plate Photopolymerization. Full details of reagents added/ removed and the procedures for distributing them are detailed in the Supporting Information. An example library synthesis is explained below. Note that the procedure for liquid monomers is slightly different from that for solid monomers.

As a representative example, to the first column of a four-column reservoir in a liquid handling robot was added 2-(dimethylamino)ethyl methacrylate $(50 \mathrm{~mL})$. To the second column of the reservoir were added 2-cyano-2-propyl dodecyltrithiocarbonate $(144 \mu \mathrm{L})$, TEOA $(9.47 \mathrm{~g})$, and methanol $(8.83 \mathrm{~mL})$. To the third column of the reservoir were added 2-cyano-2-propyl dodecyltrithiocarbonate $(103 \mu \mathrm{L})$, TEOA $(8.91 \mathrm{~g})$, and dioxane $(12.96 \mathrm{~mL})$. 2(Dimethylamino)ethyl methacrylate $(210 \mu \mathrm{L})$ was pipetted via robot to wells A1:H6, $150 \mu \mathrm{L}$ to wells $\mathrm{A} 7: \mathrm{H} 12$ of a 96 well plate, followed by $290 \mu \mathrm{L}$ of the methanol solution to wells A1:H6 and 350 $\mu \mathrm{L}$ of the dioxane solution to each of wells A7:H12 Table 1 . The plate was then covered with a TiterTop and wrapped in blue LED lights. The reaction was allowed to proceed for $24 \mathrm{~h}$, after which the lights were removed and the plate was dried under vacuum for $24 \mathrm{~h}$ to remove the solvent. The plate was then placed back into the liquid handling robot, and $1 \mathrm{~mL}$ DMF was added to each well. This plate was then agitated for $6 \mathrm{~h}$, followed by the transfer of $200 \mu \mathrm{L}$ of this solution to each well of an empty polypropylene 96-well plate. These samples were then analyzed by high-throughput SEC, and the $M_{\mathrm{p}}$ of each peak was picked out and plotted, as shown in Figure 3B.

Polymerization of NIPMAM (Solid Monomer). Into a $250 \mathrm{~mL}$ conical flask were added $15.6 \mathrm{~g}$ of $\mathrm{N}$-isopropylmethacrylamide, 18.27 $\mathrm{g}$ of triethanolamine, $27.90 \mathrm{~mL}$ of methanol, and $237 \mu \mathrm{L}$ of 2-cyano-2propyl dodecyltrithiocarbonate. This solution was mixed until it becomes homogeneous and then added to the first column of a fourcolumn reservoir. Five hundred microliters of this solution was added into each well of a 96-well deep-well plate. The plate was then covered with a TiterTop and wrapped in blue LED lights. The reaction was allowed to proceed for $24 \mathrm{~h}$ after which the lights were removed and the plate was dried under vacuum for $24 \mathrm{~h}$ to remove dioxane. The plate was then placed back into the liquid handling robot, and $1 \mathrm{~mL}$ of DMF was added to each well. This plate was then agitated for $6 \mathrm{~h}$, followed by the addition of $200 \mu \mathrm{L}$ of this solution to each well of an empty polypropylene 96-well plate. These samples were then analyzed by high-throughput SEC, and the $M_{\mathrm{p}}$ of each peak was picked out and plotted in Figure 3B.

Conversion Experiments. As a representative example, to a 20 $\mathrm{mL}$ vial were added MMA (1.01 g), TEOA (1.50 g), dioxane (2.57 $\mathrm{mL})$, cellulose triacetate (CTA, $19.5 \mu \mathrm{L})$, and DMF $(50 \mu \mathrm{L})$ and mixed until homogeneous. Five hundred microliters of this solution was pipetted into wells B2:B7 of a deep-well 96-well plate. A small sample of this stock solution was analyzed by ${ }^{1} \mathrm{H}$ NMR spectroscopy as a $t=0$ sample. The plate was then covered with a TiterTop and wrapped in blue LED lights. At indicated time points, the plate was uncovered and the content of one well was removed, followed by analysis by ${ }^{1} \mathrm{H}$ NMR spectroscopy and SEC. After $28 \mathrm{~h}$, the reaction was stopped. Conversion was determined by integration of the vinyl peaks against the DMF standard. Conversion was plotted against molecular weight, and reaction time was plotted against $\ln \left[M_{\mathrm{o}} / M_{\mathrm{n}}\right]$ to investigate the kinetics of the reaction (Figure 5).

\section{RESULTS AND DISCUSSION}

The key goal of this study was to explore the use and limits of tertiary amine additives to enable intrinsic oxygen degassing, using trithiocarbonates as iniferter/RAFT agents. Compared to current catalytic systems, this method is an easy-to-use system for high-throughput "in-air" polymer discovery that is achievable without expensive infrastructure, which is compatible with high-throughput purification to obtain polymer free of catalysts or solvents with no chromatography required. The aim is not to make the polymers with the lowest dispersities nor the "most controlled" but to have a method to speed up the synthesis, purification, and discovery processes using multiwell plates without needing specialized infrastructure that can be easily translated to a traditional RAFT polymer- 


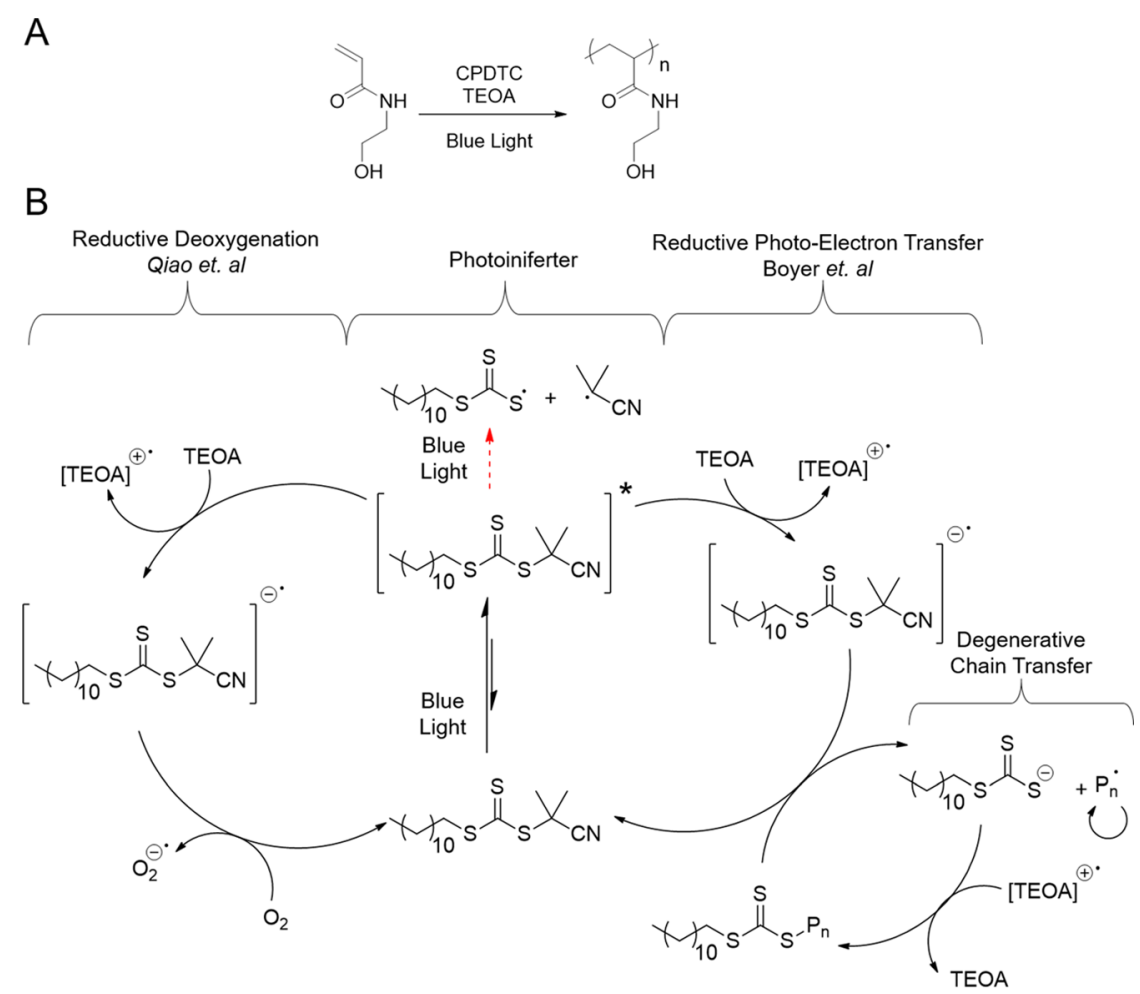

Figure 1. (A) Reaction scheme for HEA polymerizations to determine appropriate reaction volumes and TEOA concentrations. (B) Proposed mechanism for the tertiary amine-promoted deoxygenation and propagation of the reaction. Reductive deoxygenation as proposed by Qiao et al. ${ }^{39}$ and reductive photoelectron transfer as proposed by Boyer et al. ${ }^{36}$ Intense blue light may also promote photolysis of the photodegradable RAFT agent (dashed red lines) leading to an iniferter polymerization mechanism.

ization to further probe "hits" emerging from screening. The 96-well plates used are industry standard and compatible with liquid handling systems, multichannel pipettes, and highthroughput biological screening and are hence useful vessels for conducting polymerizations in. ${ }^{21,22,30}$ Conventional radical polymerization requires degassing, but there is no practical method to degas many plates at once. In addition, the degassing of the stock solutions and then inert atmosphere transfer, while possible, requires a glovebox or similar and hence presents a barrier to accessibility. To enable a simpler high-throughput method, poly(propylene) deep-well 96-well $(2 \mathrm{~mL} /$ well $)$ plates were chosen as the reaction vessels with blue light (LED) irradiation and tertiary amines, adapted from Qiao and co-workers. ${ }^{39,40}$ In all cases, no degassing of any components was employed. Pleasingly, an initial test showed the formation of polymer, proving that the system could work when open to air, but could suffer from solvent evaporation due to the heat generated by the LED lights. Therefore, reaction vessels were covered with TiterTops (a solventresistant, adhesive plastic cover) to protect the LED lights from damage caused by solvent evaporation; these are not air-tight, and the deep-well plates had significant headspace in each well. For initial tests, $N$-hydroxyethylacrylamide (HEA) was used as the monomer in dioxane with 2-cyano-2-propyl dodecyltrithiocarbonate (CPDTC) and triethanolamine (TEOA) as the tertiary amine oxygen scavengers (Figure 1 for proposed mechanisms). ${ }^{39}$ These initial conditions were chosen carefully: tertiary amine-containing monomers (e.g., dimethylaminoethylmethacrylmide) have been employed in this method, ${ }^{39,40}$ but for this work, it was important to ensure that only the additive (TEOA) could contribute to the degassing and not the monomer itself, which would increase the effective tertiary amine concentration. Dioxane was chosen as the solvent, rather than DMSO, as for library syntheses, dioxane is far easier to remove under vacuum than DMSO, vide infra. Aqueous solutions were not suitable as that would exclude hydrophobic monomers, which are important in several screening situations, such as antimicrobial materials. ${ }^{40}$

Initial polymerizations were conducted with total volumes of $0.5,1$, or $1.5 \mathrm{~mL}$ to enable the headspace effects to be probed as this has been found to be crucial in degassing ATRP/SET by reducing the rate of reoxygenation. ${ }^{44}$ Using the same stock solution for each volume, with a $[\mathrm{M}] /[\mathrm{CTA}]$ ratio of 100:1 with $[\mathrm{M}]=2 \mathrm{M}$, we saw no appreciable differences in the polymers produced (Figure S1). This suggests that total volume was not crucial and that this factor was dependent on diffusion barriers to oxygen, as has been reported by Johnson and co-workers. ${ }^{45}$ Following this, the TEOA concentration was systematically varied. The TEOA is hypothesized by Qiao to be consumed in the oxygen scavenging mechanism by enabling electron transfer to the irradiated trithiocarbonate anion, forming the oxidized species. The radical trithiocarbonate anion is then in turn oxidized through a reaction with dissolved oxygen, regenerating the trithiocarbonate and reducing the oxygen to superoxide, which is inactive and cannot terminate the polymerization, as has been shown by Boyer and others. ${ }^{39,45,46}$ Figure 1B summarizes the reactions hypothesized to occur when the trithiocarbonate is irradiated with blue light in the presence of TEOA. It can be assumed that the reductive deoxygenation pathway is favored when oxygen is present as this has been widely seen in radical polymerizations in the presence of residual oxygen and is commonly referred to as the inhibition time, that is, the time taken for any radicals generated in the system to react with oxygen after which 
propagation can occur. When the oxygen concentration is sufficiently reduced, the system will then favor the reductive photoelectron transfer pathway, leading to propagation of the polymerization in a controlled manner. In addition, it is possible that a fraction of the photodegradable RAFT agent undergoes direct photolysis, forming two radical species that would then propagate the polymerization. In a perfectly degassed system this would lead to a well-controlled polymer, but in an open system with residual oxygen, the polymerization could become uncontrolled. We hypothesize that this may be the reason for the limited molecular weight control and dispersity seen in this system (vide infra).

HEA was polymerized in a $0.5 \mathrm{~mL}$ total volume, with $[\mathrm{M}] /$ $[\mathrm{CTA}]=100: 1$ and the resulting polymers being characterized by SEC (Figure 2). Increasing the TEOA concentration from

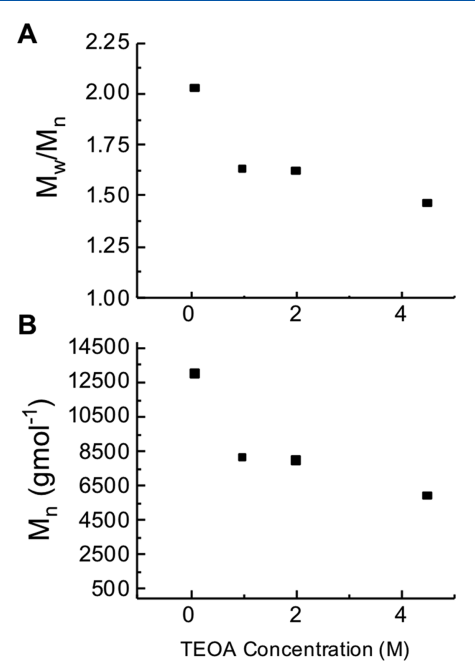

Figure 2. Polymerization of HEA at $20 \mathrm{wt} \%$ monomer using CPDTC as the iniferter and dioxane as the solvent. A $[\mathrm{M}] /[\mathrm{CTA}]$ ratio of 100:1 was used. TEOA concentration was varied, and the resulting (A) dispersity and (B) molecular weight of the polymers produced are shown.

0.22 to $4.43 \mathrm{M}$ led to a reduction in molecular weight from 13,000 to $6000 \mathrm{~g} \mathrm{~mol}^{-1}$. Figure $1 \mathrm{~B}$ shows that an increased TEOA concentration would increase the concentration of both the radical anion form of the RAFT agent and the radical cation amine. This radical cation reduces propagation by promoting conversion of the active degenerative chain transfer species to the inert form. Polymer dispersity also reduces with the addition of more TEOA (from 2.03 to $1.45 \mathrm{M}$ ), suggesting that the polymerization more closely follows the RAFT propagation/degradative chain transfer mechanism with increasing concentration of degassing agent. When no amine was present in the reaction, a small amount of very high molecular weight polymer was formed (DP > 900) likely due to an incredibly fast free-radical reaction before the diffusion of oxygen into the system caused termination and no further polymerization. This agrees with the findings of Sumerlin and co-workers, who investigated the photopolymerization mechanisms followed by trithiocarbonates under various wavelengths of irradiation, demonstrating that in a fully degassed ideal system, trithiocarbonates follow a photoiniferter mechanism under blue light. ${ }^{36}$ One hypothesis for the observation that lower dispersities are obtained with DMSO is that the superoxide irreversibly reacts with DMSO forming dimethylsulfone and would be unable to reoxidize back into triplet oxygen, which could then terminate the polymerization. In this system, more TEOA is needed to continually remove oxygen as there is no DMSO to scavenge this oxygen. ${ }^{39}$ Control experiments using $10 \%$ (v/v in final monomer, dioxane, CTA, and TEOA mixture) DMSO produced polymers with slightly lower dispersity $(\nexists=1.86)$ than the DMSO-free polymerizations $(Ð=2.12)$ but not as low as the degassed control $(\nexists=$ 1.50), suggesting DMSO provides more efficient degassing than the amine alone. However, conducting this work without DMSO is crucial to facilitate a true high-throughput approach where polymer can be easily isolated. Even higher TEOA concentrations could not be explored due to high viscosities incompatible with the liquid handling robot.

The data above confirms that pHEA can obtained in dioxane $\mathrm{BP}=101{ }^{\circ} \mathrm{C}$ ) which is easier to remove than DMSO. The dispersity values are relatively high, but it is important to highlight that this is the development of a screening tool to enable quick access to hundreds of polymers for initial screening after which conventional RAFT (or other CRP methods) can be used to study the molecular weightdependent properties of the leads in detail. To explore the library-orientated approach further, 96-well plate photopolymerizations (no degassing step) were explored using a liquid handling robot system to distribute the components. For this, the following monomers were used: dimethylaminoethyl acrylamide (DMAEMA), hydroxyethyl acrylamide (HEA), methyl acrylate (MA), and N-isopropyl acrylamide (NIPAM) with at least 48 repeats of each to determine uniformity and reproducibility of the product; it is crucial to highlight the power of this method, enabling a vast number of individual polymerization to be rapidly conducted in parallel. A TEOA concentration of $2 \mathrm{M}$ and $[\mathrm{M}] /[\mathrm{CTA}]$ ratio of 100:1 were used, using CPDTC as the iniferter with blue light irradiation for $24 \mathrm{~h}$ in the indicated solvents. To enable analysis of such a large number of samples, an SEC instrument was modified inhouse to enable sampling directly from the 96-well plates, alongside rapid SEC columns. To facilitate data analysis and reproducibility, $M_{\mathrm{p}}$ (the molecular weight at the maximum of the peak) was extracted as this could be automatically determined by a simple spreadsheet code, whereas $M_{\mathrm{n}}$ requires more extensive fitting. All SEC traces are in the Supporting Information. The results are shown in Figure 3 as both average as well as each individual polymer data points (this set alone contains 392 polymers), which is larger than most soluble polymer libraries. ${ }^{14,23}$ The solvent used had little difference on the observed molecular weights, suggesting that these are not involved in the oxygen scavenging (unlike DMSO) and that the system is not a free-radical process, where solvent has a major effect on molecular weight. To enable detailed characterization, five random samples from each monomer/ solvent combination were selected and analyzed by conventional SEC (Table S7). This confirmed that there was little variation from sample to sample. In the case of methyl acrylate, lower dispersities $(\boxplus<1.2)$ were observed than in the other polymers $(\nexists>1.5)$ most likely due to the higher rate of polymerization typical of acrylates, indicating that there was less termination due to reaction with reformed oxygen, and hence, this method might be particularly useful for acrylates. As all previous reactions had been carried out at a single $[\mathrm{M}] /$ [CTA] ratio (100:1), the ability of this technique to target varying molecular weights was investigated. A range of other monomers covering the most common types were explored (methacrylate, acrylate, acrylamide, and methacrylamide); 
A

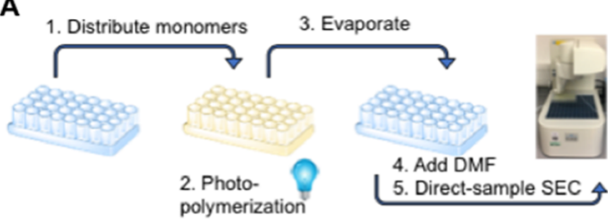

B

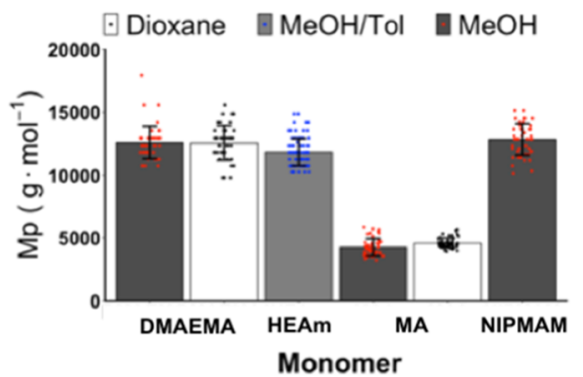

C

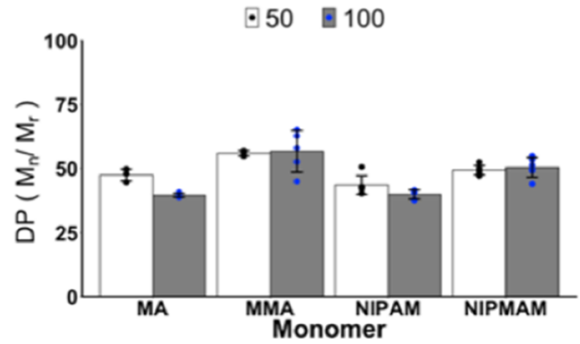

Figure 3. (A) Preparation and sampling procedure for highthroughput SEC. (B) $M_{\mathrm{p}}$ of polymers obtained using indicated solvents and monomers. (C) Obtained DP vs targeted DP (from feed ratio) for a range of monomers; $M_{r}$ is the molecular weight of the monomer. All error bars represent SD from a minimum of five repeats. Dots represent individual data points.

methyl methacrylate (MMA), MA, NIPAM, and NIPMAM were selected. Polymers were prepared using a $20 \mathrm{wt} \%$ monomer, in a $0.5 \mathrm{~mL}$ total volume, and dioxane as the solvent, with $[\mathrm{M}] /[\mathrm{CTA}]$ ratios of $100: 1$ and $50: 1$ and a final TEOA concentration of $2 \mathrm{M}$. After $24 \mathrm{~h}$ irradiation, polymers were analyzed using high-throughput SEC (Figure 3C). One sample of each was selected for further analysis on a standard SEC system (Table S8).

Across the four monomers selected, there was no difference between the molecular weights of the polymers resulting from each $[\mathrm{M}] /[\mathrm{CTA}]$ ratio tested. This lack of molecular weight control required further investigation. One method by which control could be introduced is by reducing the initiator concentration, (which in this case is also the RAFT agent) decreasing the rate of termination and therefore providing better control over the products of the reaction. However, if trithiocarbonate concentration is reduced too much, irreversible deactivation of the chain transfer agent can occur. ${ }^{47}$ Methyl methacrylate was polymerized at varying monomer concentrations from 20 to 2.5 wt \%, covering a range of initiator concentrations from 0.04 to $0.001 \mathrm{M}$. While slightly lower dispersities were observed in samples made at lower monomer concentrations, again, little molecular weight control was observed (Figure 4A and Table S9). To ensure that the initiation was not due to side reactions of another species, therefore altering the $[\mathrm{M}] /[\mathrm{I}]$ ratio and hence the molecular weight of the polymer, a control experiment with no CTA was carried out, and this afforded no polymer after $24 \mathrm{~h}$ irradiation. These findings are complimentary to Qiao's and Boyer's work, that only the efficient and irreversible removal of dissolved

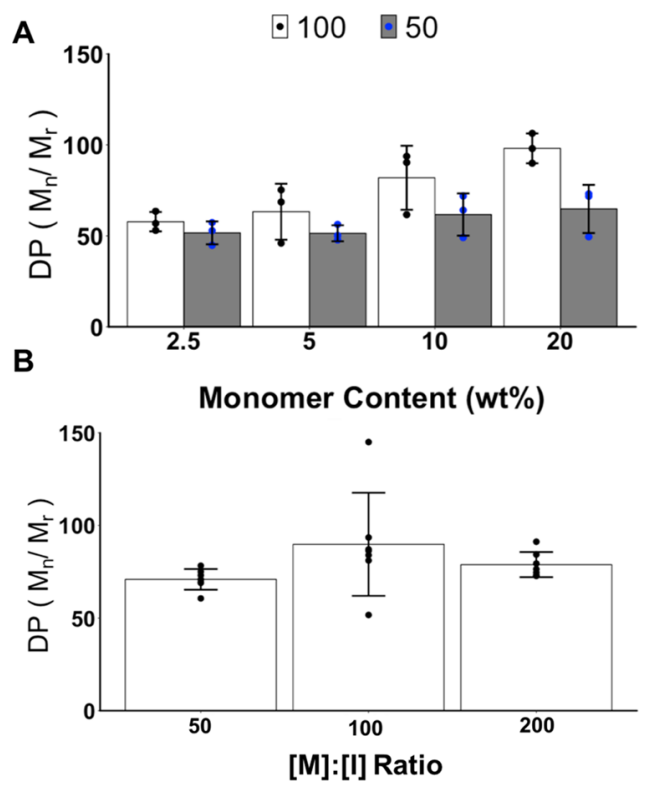

Figure 4. (A) Obtained degree of polymerization for PMMA samples made with varying monomer contents, using indicated $[\mathrm{M}] /[\mathrm{CTA}]$ ratios. Polymers were made in deep-well plates with a final TEOA concentration of $2 \mathrm{M}$, with dioxane as the solvent. (B) Obtained degree of polymerization for polymers made with a constant radical concentration. All reactions had a final CTA concentration of $0.02 \mathrm{M}$, and the monomer content was varied accordingly to target three different $[\mathrm{M}] /[\mathrm{CTA}]$ ratios. All error bars represent $\mathrm{SD}$ from a minimum of three repeats.

oxygen can enable a truly controlled/living polymerization and that a dynamic system that allows triplet oxygen to reform will always suffer from termination events.

To investigate the effects of radical concentration further, reactions with the same total radical concentration (i.e., CTA concentration) were carried out, varying the monomer concentration to target different molecular weights. This should allow for identical rates of radical generation across all targeted $[\mathrm{M}] /[\mathrm{CTA}]$ values. Again, little control over the polymerization was observed, with polymers from all three reaction conditions generating materials of similar molecular weight and dispersity (see the Supporting Information). Previous photoiniferter polymerizations of acrylates have found that using a constant radical concentration and variable monomer concentrations allowed multiple molecular weights to be accessed. These polymerizations demonstrated an initial linear character; however, deviation from this behavior was observed at higher conversions due to unidentified side reactions leading to termination. ${ }^{48}$ Photoiniferter polymerizations using NIPAM and MA have also demonstrated this two-stage kinetic process, with an initially living character up to $\sim 60 \%$ conversion after which termination reactions dominate and significantly slow down the observed molecular weight increase. ${ }^{49,50}$ These side reactions may lead to the lack of molecular weight control observed here, and therefore, the kinetics of the polymerization was investigated further.

Polymers were prepared as before, with the addition of a small amount of DMF as an internal NMR standard. Using methyl methacrylate at $20 \mathrm{wt} \%$ in dioxane at two $[\mathrm{M}] /[\mathrm{CTA}]$ ratios (100 and 50), it was found that within $24 \mathrm{~h}$, conversion had reached $90 \%$, with $99 \%$ being reached after $28 \mathrm{~h}$. This ensures that there is little monomer remaining, which is important for the polymerization of high-boiling-point 
materials as the monomer may have adverse effects on assays using material directly sampled from the plates. Kinetic plots are shown in Figure 5 and display a sharp initial increase in

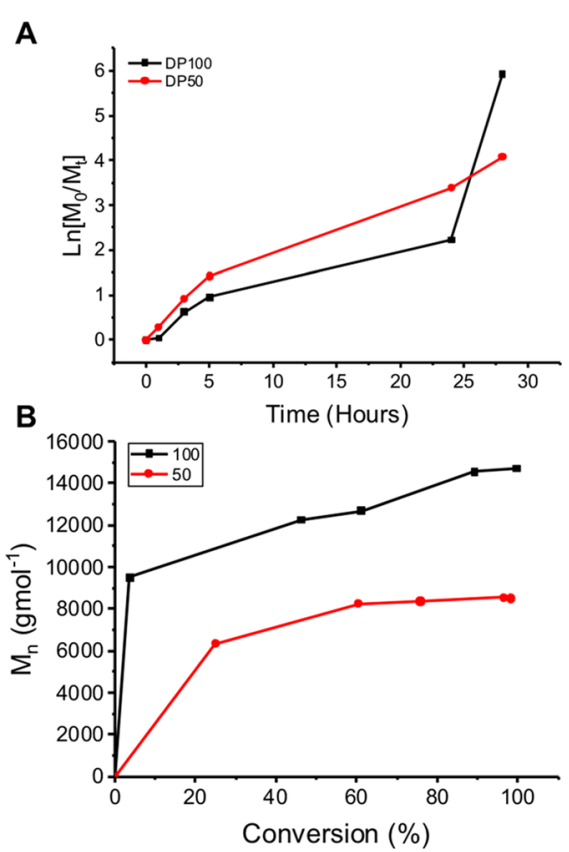

Figure 5. Kinetic plots for the polymerization of MMA at $20 \mathrm{wt} \%$ monomer in dioxane, using CPDTC as the iniferter and a final TEOA concentration of $2 \mathrm{M}$. (A) First-order kinetic plot. (B) Evolution of $M_{\mathrm{n}}$ vs conversion. $M_{\mathrm{n}}$ was determined by SEC.

molecular weight at low conversion, which suggests slow initiation (i.e., the majority of irradiated RAFT agent is initially employed converting oxygen to superoxide), and a nonlinear increase in molecular weight with conversion infers that the polymerization is not controlled. The eventual slowing of $\ln \left[M_{\mathrm{o}} / M_{\mathrm{t}}\right]$ suggests an increase in the rate of termination after $5 \mathrm{~h}$ possibly due to superoxide reverting back to the triplet state and interfering with the reaction or perhaps the loss of control associated with high conversion iniferter polymerizations discussed above. The latter is consistent with other reports of iniferter reactions in which side reactions dominate later time points, even in fully degassed systems. It is important to again note that for combinatorial approaches, full conversion (ensuring no residual monomer) is more important than stopping the reaction during the linear phase but having to then add a purification step.

The above results show that the photo-RAFT method can be used for high-throughput polymerizations of a range of monomer types without the need for DMSO, assuming that control of molecular weight by variation of [monomer]/ [RAFT agent] is not essential, which is true for many screening applications but this point must be taken into account when using this protocol. However, a key reason for moving from DMSO was to facilitate simple, in-plate, isolation of the polymers as the dialysis of hundreds of polymers is not straightforward. There is also the need to remove TEOA for most applications (although not always essential depending on the concentration required for subsequent testing ${ }^{40}$ ) to enable isolation of pure polymer. Addition of $\mathrm{HCl}$ in organic solvent precipitates the TEOA as a salt, and the 96-well plate can be centrifuged for simple isolation. Figure 6 shows ${ }^{1} \mathrm{H}$ NMR spectra of precipitated TEOA and supernatant polymer, showing essentially quantitative removal in a single step, which is again compatible with our approach of using only liquid handing at each step.

As an alternative to TEOA, triethylamine (TEA) was explored as the tertiary amine scavenger with a lower boiling point. In a trial experiment after $24 \mathrm{~h}$, MMA polymerizations in $2 \mathrm{M}$ TEA reached $80 \%$ conversion. TEA has a much lower boiling point ( 89 vs $335{ }^{\circ} \mathrm{C}$ for TEOA) and can be easily removed by drying the polymer plates under vacuum. It is important to note that due to heat generated by the lights, additional cooling may be required during this reaction to prevent evaporation of amine during the reaction. We have successfully conducted these polymerizations in a laboratory fridge or by cooling under the plate, but this is an important consideration to ensure successful polymerization. Another advantage of this process is that it also removes any solvent from the reaction allowing isolation of polymer in a single step.

\section{CONCLUSIONS}

Here, we demonstrate a useful yet easily accessible method to enable the synthesis of large polymer libraries within multiwell plates by simply using blue light and tertiary amines as intrinsic deoxygenators. Using this method, $\sim 400$ polymers were made in $<40 \mathrm{~h}$ and are easily scalable to larger libraries. The role of the tertiary amine was investigated, and it was shown that high concentrations provided polymers with a narrower molecular weight distribution. While this method uses "RAFT" agents, it is clear that this reaction has the hallmarks of an iniferter mechanism, with high degrees of termination at higher degrees of conversion. The possibility of the reaction to proceed in tandem via a reductive photoelectron transfer (PET-RAFT) mechanism further complicates any attempt at confirming a mechanism and optimizing the system to deliver predictable molecular weight distributions. Hence, control over the system in terms of predictable molecular weight from [monomer]/ [RAFT] ratios is limited, but for certain monomer classes (i.e., acrylates), low dispersity can be achieved. We therefore propose that this technique is a starting point for the synthesis of a library of polymers with varying (co)monomer compositions that do not require any complex purification for rapid screening. Once the monomer composition leads have been identified, standard living/CRP techniques can be used to investigate the effect of molecular weight on the lead (co)polymers. This approach would require far fewer polymers to be made to screen a reasonable molecular weight range and would be suitable for many biological screening applications where monomer composition of the polymer usually is the primary differentiator of function. The reproducibility of the method was tested by running $>40$ repeats of each monomer/ solvent combination. Using a modified plate-sampling SEC system, the variance was shown to be small, and the position of the reaction in the 96-well plate had no effect on its outcome, which is crucial for screening. Dioxane was found to be a suitable solvent, with the advantage that it is easy to remove by simple evaporation at the end of the reaction. It was also shown how the tertiary amines (triethanolamine and triethylamine) can be removed from the product using a single-step purification in-plate enabling isolation of pure polymers, which is not easily achieved by other in-plate methods. The power of this method lies in its simplicity, rapidity, and scalability to enable libraries of differently functional polymers to emerge to screen for function. 


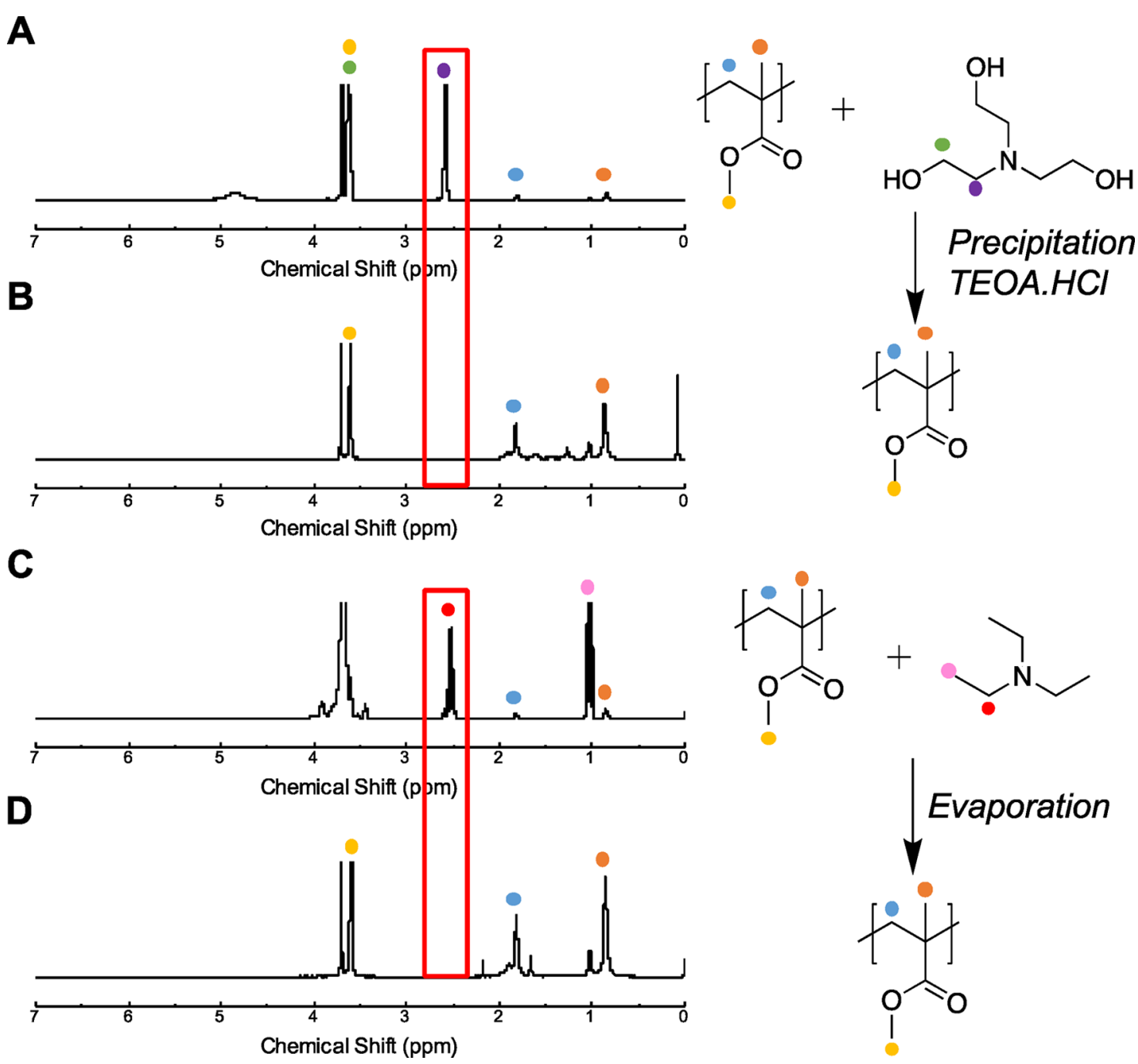

Figure 6. Oxygen scavenger removal demonstrated by ${ }^{1} \mathrm{H}$ NMR spectroscopy $\left(\mathrm{CDCl}_{3}\right)$. (A) PMMA and TEOA; (B) PMMA and TEOA supernatant after the addition of $\mathrm{HCl}$; (C) PMMA and TEA; and (D) PMMA and TEA after drying under vacuum for $24 \mathrm{~h}$. Peak at 3.70 ppm is the residual dioxane. The highlighted area (red box) shows the region in which the characteristic $\left(\mathrm{CH}_{2}\right)_{3} \mathrm{~N}$ peak is located.

\section{ASSOCIATED CONTENT}

\section{S Supporting Information}

The Supporting Information is available free of charge on the ACS Publications website at DOI: 10.1021/acs.macromol.9b01714.

Experimental procedures and characterization data, including size exclusion chromatography traces (PDF)

\section{AUTHOR INFORMATION}

\section{Corresponding Author}

*E-mail: M.I.Gibson@warwick.ac.uk.

ORCID $\odot$

Matthew I. Gibson: 0000-0002-8297-1278

\section{Author Contributions}

The manuscript was written through contributions of all authors. All authors have given approval to the final version of the manuscript.

\section{Notes}

The authors declare no competing financial interest.

The research data supporting this publication can be found at http://wrap.warwick.ac.uk.

\section{ACKNOWLEDGMENTS}

MIG holds an ERC starting grant (CRYOMAT 638661) and ERC PoC grant (789182). The Warwick Polymer Research Technology Platform is acknowledged for the SEC analysis. JD is funded by the MRC Doctoral Training Partnership (grant no. MR/N014294/1). BBSRC and InnovateUK are also acknowledged for grant BB/M02878X/1.

\section{REFERENCES}

(1) Boyer, C.; Bulmus, V.; Davis, T. P.; Ladmiral, V.; Liu, J.; Perrier, S. Bioapplications of RAFT Polymerization. Chem. Rev 2009, 109, $5402-5436$.

(2) Cobo, I.; Li, M.; Sumerlin, B. S.; Perrier, S. Smart Hybrid Materials by Conjugation of Responsive Polymers to Biomacromolecules. Nat. Mater. 2015, 14, 143-159.

(3) Biggs, C. I.; Bailey, T. L.; Graham, B.; Stubbs, C.; Fayter, A.; Gibson, M. I. Polymer Mimics of Biomacromolecular Antifreezes. Nat. Commun. 2017, 8, 1546.

(4) Lutz, J.-F. An Introduction to Sequence-Controlled Polymers. In Sequence-Controlled Polymers: Synthesis, Self-Assembly, and Properties; Meyer, T. Y.; Ouchi, M.; Sawamoto, M., Eds.; American Chemical Society: Washington, DC, 2014; Vol. 1170, p 1.

(5) Zydziak, N.; Konrad, W.; Feist, F.; Afonin, S.; Weidner, S.; Barner-Kowollik, C. Coding and Decoding Libraries of Sequence- 
Defined Functional Copolymers Synthesized via Photoligation. Nat. Commun. 2016, 7, 13672.

(6) Martens, S.; Van Den Begin, J.; Madder, A.; Du Prez, F. E.; Espeel, P. Automated Synthesis of Monodisperse Oligomers, Featuring Sequence Control and Tailored Functionalization. J. Am. Chem. Soc. 2016, 138, 14182-14185.

(7) Gody, G.; Barbey, R.; Danial, M.; Perrier, S. Ultrafast RAFT Polymerization: Multiblock Copolymers within Minutes. Polym. Chem. 2015, 6, 1502-1511.

(8) Engelis, N. G.; Anastasaki, A.; Nurumbetov, G.; Truong, N. P.; Nikolaou, V.; Shegiwal, A.; Whittaker, M. R.; Davis, T. P.; Haddleton, D. M. Sequence-Controlled Methacrylic Multiblock Copolymers via Sulfur-Free RAFT Emulsion Polymerization. Nat. Chem. 2017, 9, 171-178.

(9) Ting, J. M.; Porter, W. W., III; Mecca, J. M.; Bates, F. S.; Reineke, T. M. Advances in Polymer Design for Enhancing Oral Drug Solubility and Delivery. Bioconjugate Chem. 2018, 29, 939-952.

(10) Anderson, D. G.; Lynn, D. M.; Langer, R. Semi-Automated Synthesis and Screening of a Large Library of Degradable Cationic Polymers for Gene Delivery. Angew. Chem., Int. Ed. 2003, 42, 31533158.

(11) Mei, Y.; Saha, K.; Bogatyrev, S. R.; Yang, J.; Hook, A. L.; Kalcioglu, Z. I.; Cho, S. W.; Mitalipova, M.; Pyzocha, N.; Rojas, F.; Van Vliet, K. J.; Davies, M. C.; Alexander, M. R.; Langer, R.; Jaenisch, R.; Anderson, D. G. Combinatorial Development of Biomaterials for Clonal Growth of Human Pluripotent Stem Cells. Nat. Mater. 2010, 9, 768-778.

(12) Greenaway, R. L.; Santolini, V.; Bennison, M. J.; Alston, B. M.; Pugh, C. J.; Little, M. A.; Miklitz, M.; Eden-Rump, E. G. B.; Clowes, R.; Shakil, A.; Cuthbertson, H. J.; Armstrong, H.; Briggs, M. E.; Jelfs, K. E.; Cooper, A. I. High-Throughput Discovery of Organic Cages and Catenanes Using Computational Screening Fused with Robotic Synthesis. Nat. Commun 2018, 9, 2849.

(13) Bray, C. L.; Tan, B.; Wood, C. D.; Cooper, A. I. HighThroughput Solubility Measurements of Polymer Libraries in Supercritical Carbon Dioxide. J. Mater. Chem. 2005, 15, 456-459.

(14) Haven, J. J.; Guerrero-Sanchez, C.; Keddie, D. J.; Moad, G.; Thang, S. H.; Schubert, U. S. One Pot Synthesis of Higher Order Quasi-Block Copolymer Libraries via Sequential RAFT Polymerization in an Automated Synthesizer. Polym. Chem. 2014, 5, 52365246.

(15) Chapon, P.; Mignaud, C.; Lizarraga, G.; Destarac, M. Automated Parallel Synthesis of MADIX (Co)Polymers. Macromol. Rapid Commun. 2003, 24, 87-91.

(16) Zhang, H.; Fijten, M. W. M.; Hoogenboom, R.; Reinierkens, R.; Schubert, U. S. Application of a Parallel Synthetic Approach in AtomTransfer Radical Polymerization: Set-up and Feasibility Demonstration. Macromol. Rapid Commun. 2003, 24, 81-86.

(17) Hoogenboom, R.; Fijten, M. W. M.; Meier, M. A. R.; Schubert, U. S. Living Cationic Polymerizations Utilizing an Automated Synthesizer : High-Throughput Synthesis of Polyoxazolines. Macromol. Rapid Commun. 2003, 92-97.

(18) Hoogenboom, R.; Schubert, U. S. The Fast and the curious: High-Throughput Experimentation in synthetic polymer chemistry. J. Polym. Sci., Part A: Polym. Chem. 2003, 41, 2425-2434.

(19) Hook, A. L.; Chang, C. Y.; Yang, J.; Luckett, J.; Cockayne, A.; Atkinson, S.; Mei, Y.; Bayston, R.; Irvine, D. J.; Langer, R.; Anderson, D. G.; Williams, P.; Davies, M. C.; Alexander, M. R. Combinatorial Discovery of Polymers Resistant to Bacterial Attachment. Nat. Biotechnol. 2012, 30, 868-875.

(20) Ting, J. M.; Tale, S.; Purchel, A. A.; Jones, S. D.; Widanapathirana, L.; Tolstyka, Z. P.; Guo, L.; Guillaudeu, S. J.; Bates, F. S.; Reineke, T. M. High-Throughput Excipient Discovery Enables Oral Delivery of Poorly Soluble Pharmaceuticals. ACS Cent. Sci. 2016, 2, 748-755.

(21) Weissleder, R.; Kelly, K.; Sun, E. Y.; Shtatland, T.; Josephson, L. Cell-Specific Targeting of Nanoparticles by Multivalent Attachment of Small Molecules. Nat. Biotechnol. 2005, 23, 1418-1423.
(22) Janzen, W. P. Screening Technologies for Small Molecule Discovery: The State of the Art. Chem. Biol. 2014, 21, 1162-1170.

(23) Chapman, R.; Gormley, A. J.; Herpoldt, K.-L.; Stevens, M. M. Highly Controlled Open Vessel RAFT Polymerizations by Enzyme Degassing. Macromolecules 2014, 47, 8541-8547.

(24) Chapman, R.; Gormley, A. J.; Stenzel, M. H.; Stevens, M. M. Combinatorial Low-Volume Synthesis of Well-Defined Polymers by Enzyme Degassing. Angew. Chem., Int. Ed. 2016, 55, 4500-4503.

(25) Schneiderman, D. K.; Ting, J. M.; Purchel, A. A.; Miranda, R., Jr.; Tirrell, M. V.; Reineke, T. M.; Rowan, S. J. Open-to-Air RAFT Polymerization in Complex Solvents: From Whisky to Fermentation Broth. ACS Macro Lett. 2018, 7, 406-411.

(26) Enciso, A. E.; Fu, L.; Russell, A. J.; Matyjaszewski, K. A Breathing Atom-Transfer Radical Polymerization: Fully OxygenTolerant Polymerization Inspired by Aerobic Respiration of Cells. Angew. Chem., Int. Ed. January 22, 2018, 933-936.

(27) Fors, B. P.; Hawker, C. J. Control of a Living Radical Polymerization of Methacrylates by Light. Angew. Chem., Int. Ed. 2012, 51, 8850-8853.

(28) Xu, J.; Shanmugam, S.; Duong, H. T.; Boyer, C. OrganoPhotocatalysts for Photoinduced Electron Transfer-Reversible Addition-Fragmentation Chain Transfer (PET-RAFT) Polymerization. Polym. Chem. 2015, 6, 5615-5624.

(29) Gormley, A. J.; Yeow, J.; Ng, G.; Conway, Ó.; Boyer, C.; Chapman, R. An Oxygen-Tolerant PET-RAFT Polymerization for Screening Structure-Activity Relationships. Angew. Chem., Int. Ed. 2018, 57, 1557-1562.

(30) Yeow, J.; Chapman, R.; Xu, J.; Boyer, C. Oxygen Tolerant Photopolymerization for Ultralow Volumes. Polym. Chem. 2017, 8, 5012-5022.

(31) Shanmugam, S.; Xu, J.; Boyer, C. Photoinduced Electron Transfer-Reversible Addition-Fragmentation Chain Transfer (PETRAFT) Polymerization of Vinyl Acetate and N-Vinylpyrrolidinone: Kinetic and Oxygen Tolerance Study. Macromolecules 2014, 47, 4930-4942.

(32) Oliver, S.; Zhao, L.; Gormley, A. J.; Chapman, R.; Boyer, C. Living in the Fast Lane - High Throughput Controlled/Living Radical Polymerization. Macromolecules 2019, 27, 3-23.

(33) Blackman, L. D.; Varlas, S.; Arno, M. C.; Fayter, A.; Gibson, M. I.; O’Reilly, R. K. Permeable Protein-Loaded Polymersome Cascade Nanoreactors by Polymerization-Induced Self-Assembly. ACS Macro Lett. 2017, 6, 1263-1267.

(34) Blackman, L. D.; Varlas, S.; Arno, M. C.; Houston, Z. H.; Fletcher, N. L.; Thurecht, K. J.; Hasan, M.; Gibson, M. I.; O’Reilly, R. K. Confinement of Therapeutic Enzymes in Selectively Permeable Polymer Vesicles by Polymerization-Induced Self-Assembly (PISA) Reduces Antibody Binding and Proteolytic Susceptibility. ACS Cent. Sci. 2018, 4, 718-723.

(35) Tucker, B. S.; Coughlin, M. L.; Figg, C. A.; Sumerlin, B. S. Grafting-From Proteins Using Metal-Free PET-RAFT Polymerizations under Mild Visible-Light Irradiation. ACS Macro Lett. 2017, 6, 452-457.

(36) Figg, C. A.; Hickman, J. D.; Scheutz, G. M.; Shanmugam, S.; Carmean, R. N.; Tucker, B. S.; Boyer, C.; Sumerlin, B. S. ColorCoding Visible Light Polymerizations To Elucidate the Activation of Trithiocarbonates Using Eosin Y. Macromolecules 2018, 51, 13701376.

(37) Stubbs, C.; Congdon, T. R.; Gibson, M. I. Photo-Polymerisation and Study of the Ice Recrystallisation Inhibition of Hydrophobically Modified Poly(Vinyl Pyrrolidone) Co-Polymers. Eur. Polym. J. 2019, 110, 330-336.

(38) Blackman, L. D.; Doncom, K. E. B.; Gibson, M. I.; O’Reilly, R. K. Comparison of Photo- and Thermally Initiated PolymerizationInduced Self-Assembly: A Lack of End Group Fidelity Drives the Formation of Higher Order Morphologies. Polym. Chem. 2017, 8, 2860-2871.

(39) Fu, Q.; Xie, K.; McKenzie, T. G.; Qiao, G. G. Trithiocarbonates as Intrinsic Photoredox Catalysts and RAFT Agents for Oxygen 
Tolerant Controlled Radical Polymerization. Polym. Chem. 2017, 8, $1519-1526$.

(40) Richards, S.-J.; Jones, A.; Tomás, R. M. F.; Gibson, M. I. Photochemical "In-Air" Combinatorial Discovery of Antimicrobial Copolymers. Chem. - Eur. J. 2018, 24, 13758-13761.

(41) Pal, R.; Mamidi, M. K.; Das, A. K.; Bhonde, R. Diverse Effects of Dimethyl Sulfoxide (DMSO) on the Differentiation Potential of Human Embryonic Stem Cells. Arch. Toxicol. 2012, 86, 651-661.

(42) Thaler, R.; Spitzer, S.; Karlic, H.; Klaushofer, K.; Varga, F. DMSO Is a Strong Inducer of DNA Hydroxymethylation in PreOsteoblastic MC3T3-E1 Cells. Epigenetics 2012, 7, 635-651.

(43) Yi, X.; Liu, M.; Luo, Q.; Zhuo, H.; Cao, H.; Wang, J.; Han, Y. Toxic Effects of Dimethyl Sulfoxide on Red Blood Cells, Platelets, and Vascular Endothelial Cells in Vitro. FEBS Open Bio 2017, 7, 485-494.

(44) Liarou, E.; Whitfield, R.; Anastasaki, A.; Engelis, N. G.; Jones, G. R.; Velonia, K.; Haddleton, D. M. Copper-Mediated Polymerization without External Deoxygenation or Oxygen Scavengers. Angew. Chem., Int. Ed. 2018, 57, 8998-9002.

(45) Lamb, J. R.; Qin, K. P.; Johnson, J. A. Visible-Light Mediated, Additive-Free, and Open-to-Air Controlled Radical Polymerization of Acrylates and Acrylamides. Polym. Chem. 2019, 10, 1585.

(46) Ouannes, C.; Wilson, T. Quenching of Singlet Oxygen by Tertiary Aliphatic Amines. Effect of DABCO (1,4-diazabicyclo[2.2.2]octane). J. Am. Chem. Soc. 1968, 90, 6527-6528.

(47) Wang, H.; Li, Q.; Dai, J.; Du, F.; Zheng, H.; Bai, R. Real-Time and in Situ Investigation of "Living"/Controlled Photopolymerization in the Presence of a Trithiocarbonate. Macromolecules 2013, 46, $2576-2582$.

(48) Lambrinos, P.; Tardi, M.; Polton, A.; Sigwalt, P. The Mechanism of the Polymerization of n.Butyl Acrylate Initiated with N,N-Diethyl Dithiocarbamate Derivatives. Eur. Polym. J. 1990, 26, $1125-1135$.

(49) Ishizu, K.; Khan, R. A.; Furukawa, T.; Furo, M. Controlled Radical Polymerization of N-Isopropylacrylamide Initiated by Photofunctional 2-(N,N-Diethyldithiocarbamyl)Isobutyric Acid Sodium Salt in Aqueous Medium. J. Appl. Polym. Sci. 2004, 91, 3233-3238.

(50) Ishizu, K.; Katsuhara, H.; Itoya, K. Controlled Radical Polymerization of Methacrylic Acid Initiated by DiethyldithioCarbamate-Mediated Iniferter. J. Polym. Sci., Part A Polym. Chem. 2005, 43, 230-233. 